Article

\title{
Nondestructive Internal Defect Detection Using a CW-THz Imaging System in XLPE for Power Cable Insulation
}

\author{
In-Sung Lee and Joong Wook Lee * \\ Department of Physics and Optoelectronics Convergence Research Center, Chonnam National University, \\ Gwangju 61186, Korea; mh870214@gmail.com \\ * Correspondence: leejujc@chonnam.ac.kr
}

Received: 10 February 2020; Accepted: 11 March 2020; Published: 18 March 2020

check for updates

\begin{abstract}
The demand for internal nondestructive testing and inspection techniques is rapidly increasing. Using a continuous wave $(\mathrm{CW})$ terahertz $(\mathrm{THz})$ imaging system, we demonstrate that the internal defects in cross-linked polyethylene (XLPE) plates for power cable insulation can be detected. In a coherent detection scheme based on photomixers, which serve as the $\mathrm{THz}$ emitters and receivers, the change of phase occurring with the defects inside the XLPE plates is distinctly measured by the change in the amplitude of the transmitted $\mathrm{THz}$ waves. According to the two-dimensional images of $\mathrm{THz}$ waves transmitted through the XLPE plates, defects of up to $0.5 \mathrm{~mm}$ size located inside the XLPE plates can be detected by the internal nondestructive examination method based on CW-THz waves. This technique will be useful for internal nondestructive testing and inspection of insulation materials that require high resolution in various industries, including the automobiles, electronics, and electrical power industries.
\end{abstract}

Keywords: nondestructive examination; XLPE insulation; terahertz imaging; power cable

\section{Introduction}

An electrical power system is generally considered as a large network that connects power plants to customers; it consists of electricity generators and electrical power transmission and distribution networks. One of the most important components in electric power systems is insulators for high-voltage power cables. Cross-linked polyethylene (XLPE), which is a form of polyethylene with crosslinks, is widely used as an insulation material because of its excellent advantages, including high resistance against weather, moisture, and temperature, high mechanical strength, and remarkable electrical properties [1,2].

All dielectrics, including XLPE, undergo several alterations in their physical or chemical properties as a result of internal or external environmental factors, e.g., mechanical constraints, humidity, temperature, and partial discharges. Aging can lead to the dielectric breakdown of power cables with XLPE insulation and increase the instability of the electric power system [3-6]. Therefore, the study of the aging of XLPE is becoming increasingly important. In the macroscopic regime, measurements of space charge, insulation resistance, and temperature have been applied for detecting the characteristics of the aging of XLPE, whereas in the microscopic regime, X-ray diffraction has been typically used to characterize XLPE insulation [7]. Above all, it is important to study the influence of defects on the aging phenomenon and the degradation of the insulating performance. The partial discharges induced by defects in the XLPE insulation usually reduce insulation performance in the long term and can act as a factor that further impedes the stability and safety of the overall electric power system. Measurements of partial discharges have been used in one important way to detect defects in XLPE insulation [8]. 
Recently, terahertz $(\mathrm{THz})$ spectroscopy and imaging techniques have attracted attention as new methods for studying insulation materials, as well as for realizing nondestructive testing and inspection [9-14]. In particular, the photon energy of $\mathrm{THz}$ waves, e.g., $4.1 \mathrm{meV}$ at $1 \mathrm{THz}$, corresponds to the energy range of intermolecular interactions, lattice vibration, and molecular motions such as vibration and rotation. This property enables the investigation of aging effects in XLPE using THz time-domain spectroscopy [15-18]. More importantly, THz techniques can be applied to detect defects inside XLPE insulation. Defects such as voids and conducting particles in XLPE insulation can lead to strong and inhomogeneous electric fields around the defects, internal discharges such as treeing discharge, and acceleration of the aging process in polymeric insulated power cables, in order. Detecting the defects using an internal nondestructive examination method is thus particularly important. In addition, the advantage of continuous-wave (CW)-THz imaging systems is that they are able to work contactless and target insulators with extremely low electrical conductivity. Therefore, $\mathrm{THz}$ technologies can provide testing methods safer than existing methods that need to get the electrodes in contact such as electrical impedance tomography [19], or cannot be used for detecting defects in power cables such as Eddy-current testing [20]. Furthermore, the THz imaging system is expected to be an easy and simple testing method, so it does not require any careful attention by experienced technicians, unlike ultrasonic testing [21]. In this paper, we used a CW THz imaging system as a nondestructive examination tool for detecting void defects inside XLPE insulation.

\section{Materials and Methods}

$\mathrm{CW}-\mathrm{THz}$ radiation was generated by an optical heterodyning method using two distributed feedback (DFB) diode lasers (laser 1 and laser 2 with wavelengths $\lambda_{1}$ and $\lambda_{2}$, respectively; Figure 1a) at $1550 \mathrm{~nm}$. The beat signals, which happen to be the frequency difference, $\Delta \lambda=\lambda_{1}-\lambda_{2}$, between the two lasers, are divided into two parts and illuminate both the transmitter and receiver simultaneously. The photomixer that serves as the THz transmitter is based on an InGaAs/InP semiconductor structure with a bandgap of approximately $1.5 \mu \mathrm{m}$. When applying a bias voltage to the metal electrodes deposited on the surface of the $\mathrm{THz}$ transmitter, the beat signals generate a photocurrent that oscillates at its beat frequency and is then converted into $\mathrm{THz}$ radiation.
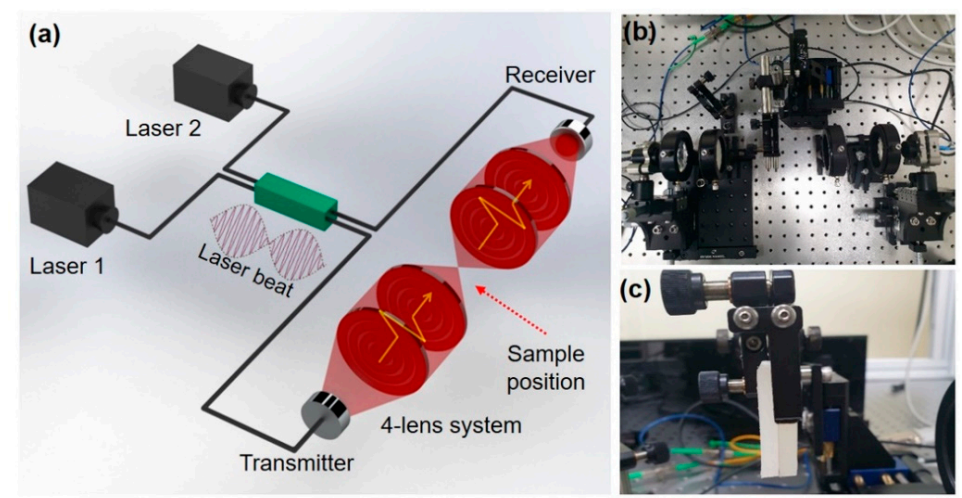

Figure 1. (a) Schematic of continuoius wave (CW)-terahertz (THz) imaging system, which consists of two distributed feedback (DFB) diode lasers, a fiber splitter, two photomixers that serve as the $\mathrm{THz}$ transmitter and receiver, four THz lenses, and a twoaxis motorized sample stage. (b) Photograph of the $\mathrm{CW}-\mathrm{THz}$ imaging system. (c) Photograph of the sample mount and the sample with artificial void defects inside.

The generated $\mathrm{THz}$ waves are focused at the center of the four-THz-lens system, at which point the samples are placed, as shown in Figure 1a,b. Both the transmitted $\mathrm{THz}$ waves and the beat signals illuminate the photomixer acting as the $\mathrm{THz}$ receiver. The beat signals generate photo-carriers, and the amplitude of the electric field of incident $\mathrm{THz}$ waves induces the resulting photocurrent. Measurements using the $\mathrm{THz}$ receiver, therefore, depend on the phase difference between the beat signal and the 
incident $\mathrm{THz}$ wave. This means that the $\mathrm{THz}$ technique shown in Figure 1 is a coherent detection method that has the advantages of very high efficiency and sensitivity.

In our experiments, the void defects inside the XLPE plates cause a change in the optical path length, compared with the area filled with the XLPE material. The change in phase caused by the difference in optical path length between the defects, and the XLPE material surrounding them corresponds to the change in the amplitude of the measured transmission of the $\mathrm{THz}$ waves in the coherent detection scheme. For example, if there is a void defect with $0.3 \mathrm{~mm}$ depth in XLPE whose refractive index is around 1.5 at $\mathrm{THz}$ frequencies, the difference of optical path lengths between the transmission signals passing through the void defect point and the surrounding material without any void defect is $0.15 \mathrm{~mm}$ and the phase difference is $\pi$ at the frequency of $1 \mathrm{THz}$. In that case, the direction of photocurrent induced by the $\mathrm{THz}$ waves incident on the surface of the $\mathrm{THz}$ receive becomes completely reversed and thus the magnitude values of transmitted $\mathrm{THz}$ electric field are the same despite that the signs are opposite. Therefore, it is possible to extract a scan image of the samples by measuring the signal size of the transmitted $\mathrm{THz}$ waves at each point while moving the XLPE block containing the internal defects along two axes.

To realize an XLPE block sample with artificial void defects inside, we first cut a XLPE plate with a thickness of approximately $10 \mathrm{~mm}$ into two pieces of half the total thickness each. On the inner surface of one of the two pieces, artificial void defects of cylindrical shape specifically designed for our experiments were created using a femtosecond laser micromachining method, which is based on laser ablation by amplified femtosecond laser pulses [22]. Ultrashort laser pulses enable the fabrication of machined structures with high quality, as shown in Figure 2a, because the duration of laser pulses less than the conduction time of materials reduces the heat effect and minimizes debris. When assembling the two split pieces together, the fabricated void defects were placed inside the XLPE block, as shown in Figure 1c.
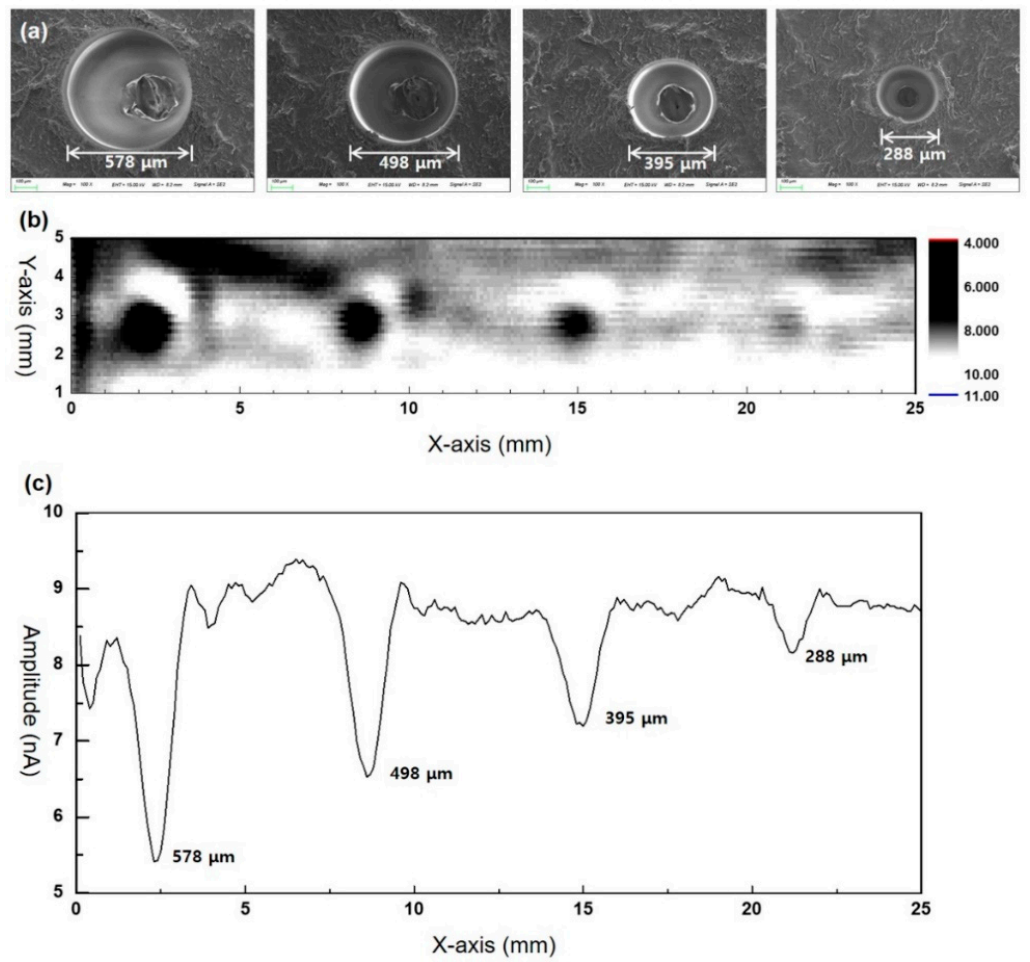

Figure 2. (a) Scanning electron micrographs of the artificial void defects with diameters of 578, 498, 395, and $288 \mu \mathrm{m}$. The depth of the defects is $1.2 \mathrm{~mm}$, and the distance between the defects is approximately $6 \mathrm{~mm}$. (b) The two-dimensional amplitude image of transmitted CW-THz waves with $1 \mathrm{THz}$ frequency. (c) Cross-sectional profile along the $x$-axis at the center of the amplitude dips that originate from the artificial void defects. 


\section{Results and Discussion}

Figure 2a shows scanning electron microscopy images of the artificial void defects fabricated by the laser machining method. For each distance of approximately $6 \mathrm{~mm}$, four side-by-side defects with diameters of 578, 498, 395, and $288 \mu \mathrm{m}$, and a depth of $1.2 \mathrm{~mm}$, were formed inside an XLPE plate with a thickness of approximately $10 \mathrm{~mm}$.

Figure $2 \mathrm{~b}$ shows the two-dimensional amplitude image of the $\mathrm{THz}$ waves transmitted through the sample. Because we optimized the transmission signal in the defect-free bulk XLPE plate, the change in the transmitted signal resulting from the void defects appears as dips, as shown by the dark circles in Figure $2 \mathrm{~b}$. The image pinpoints the location of the void defects. Figure $2 \mathrm{c}$ also shows the cross-sectional profile along the $x$-axis at the center of the amplitude dips for the four void defects. The results show that not only is identification of a very small defect with a $288-\mu \mathrm{m}$ diameter possible, but also that extracting information regarding the shape and size of each defect is possible using the CW-THz imaging system.

The value of the full width at half maximum (FWHM) and the depth of signal amplitude for four artificial void defects with various diameters, as shown in Figure 3, show steadily rising curves as the diameter increases. As the diameter of the defect increases, so does the depth of transmission amplitude, and the easier it is to distinguish the defects from the surrounding XLPE material. Even in the smallest defect, with a diameter of $288 \mu \mathrm{m}$, the value of amplitude depth, approximately $0.8 \mathrm{nA}$, is quite distinguishable against the adjacent area, showing a deviation of up to $0.5 \mathrm{nA}$. The values of FWHM also exhibit a similar trend with respect to the diameter of the defects.

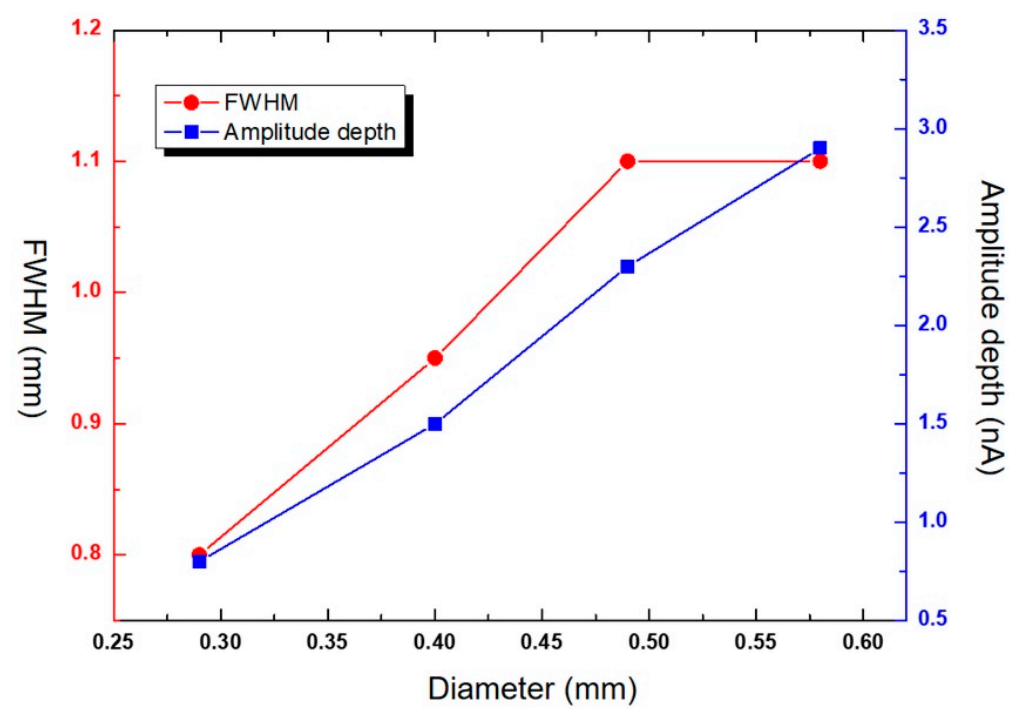

Figure 3. Full width at half maximum (FWHM) (red circles) and the depth of signal amplitude through the artificial void defects with various diameters (blue squares). The double y axes are used for comparing the two data sets, i.e., the FWHM values and amplitude depths.

To verify the resolution of the CW-THz imaging system used in the experiment, samples with two adjacent void defects with various diameters and gap widths were produced, as shown in Figure 4a. The samples were designed to have diameters of 600,500,400, and $300 \mu \mathrm{m}$ and gap widths of 500,400, 300 , and $200 \mu \mathrm{m}$, respectively. The actual measured values of the diameter and gap width are shown in Figure $4 a, b$, which shows the two-dimensional scanning image of the sample and contains all four structures (denoted S1, S2, S3, and S4). The cross-sectional profile of the transmitted amplitude signal along the $x$-axis at the center of the dips more clearly reveals the shape of the artificial void defects, as shown in Figure 4c. 
(a)
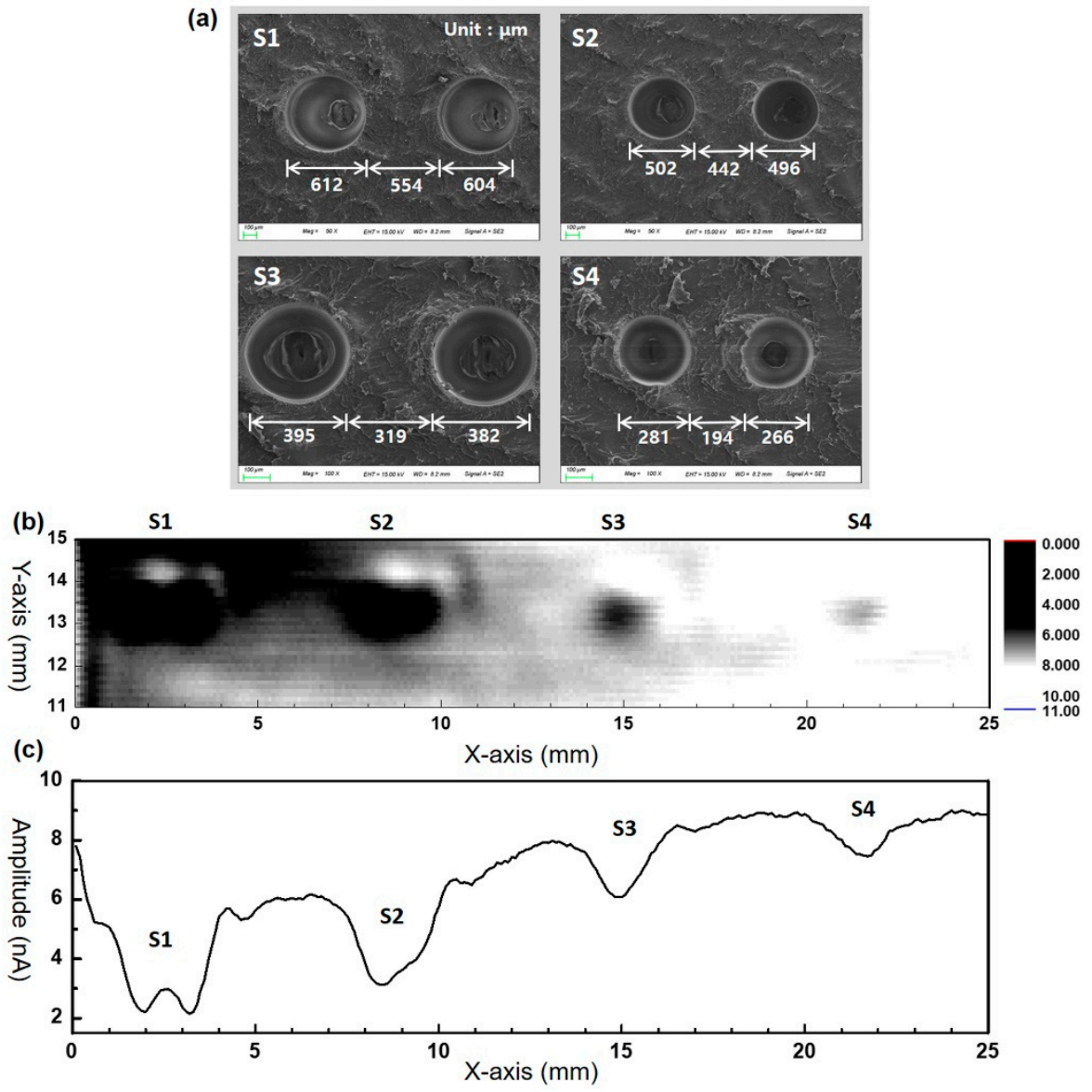

Figure 4. (a) Scanning electron micrographs (SEM) of double-void defects with various diameters and gap widths (diameter-gap width-diameter: 612-554-604 $\mu \mathrm{m}$; 502-442-496 $\mu \mathrm{m}$; 395-319-382 $\mu \mathrm{m}$; and 281-194-266 $\mu \mathrm{m}$, denoted S1, S2, S3, and S4, respectively). The depths of the defects are $1.2 \mathrm{~mm}$. For the sake of clarity, the unit lengths in the upper and lower figures are different. (b) Two-dimensional amplitude image of transmitted CW-THz waves with $1 \mathrm{THz}$ frequency. (c) Cross-sectional profile along the $x$-axis at the center of the amplitude dips that originate from the four double-void defects.

For the double-void defect with a diameter of approximately $600 \mu \mathrm{m}, \mathrm{S} 1$, the two defects seem to be clearly distinguishable. In addition, in the case of $500 \mu \mathrm{m}, \mathrm{S} 2$, at first glance, it looks like only one dip is observable but there seems to be another small dip on the right shoulder of the main dip. On the contrary, in the case of two structures with diameters of approximately 400 and $300 \mu \mathrm{m}$, it is difficult to distinguish the two adjacent void defects. This means that the CW-THz imaging system using a frequency of $1 \mathrm{THz}$ offers a sufficiently distinct resolution up to approximately $500 \mu \mathrm{m}$ for two adjacent defects.

Three-dimensional plots showing the signal amplitude around the double-void defects and the results of double Gaussian fitting of the cross-sectional profiles along the center of defects for S1 and S2 are shown in Figure 5. For the sake of convenient data analysis, the $y$-axis is flipped so that the signals from the defects become peaks. To model the contrast profile of the defect points, we represent the double-void defect signals as the sum of cylindrically symmetric Gaussian peaks, based on the double Gaussian fitting method. 

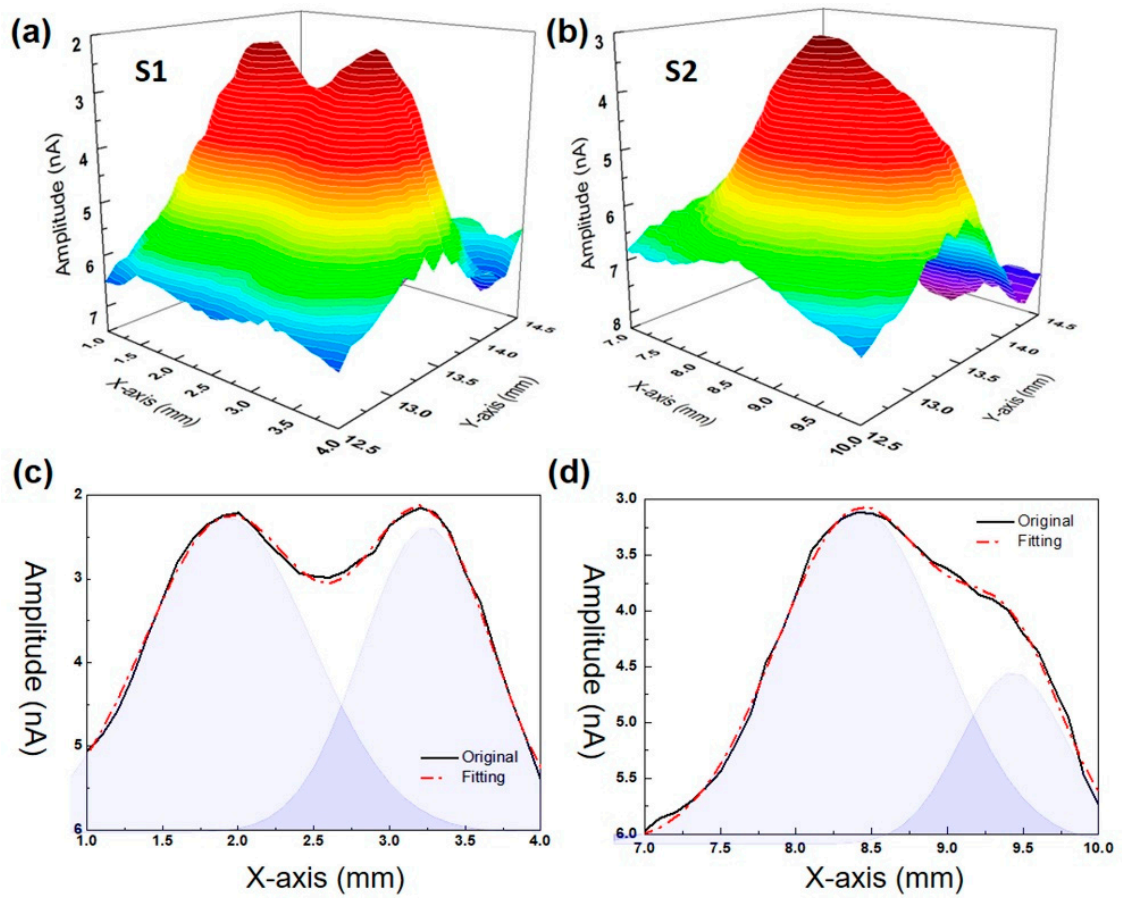

Figure 5. (a,b) Three-dimensional plots near the double-void defects of S1 and S2, respectively. (c,d) Cross-sectional profiles along the $x$-axis at the center of the amplitude dips for S1 and S2 (black solid curves) and the double Gaussian fittings (red dot-dashed curves). The amplitude curves are well fitted by the double Gaussian components that are plotted with filled area two-dimensional plots (the areas that appear in purple).

For S1, the center-to-center distance between the two Gaussian peaks is $1.32 \mu \mathrm{m}$, which corresponds to the actual value of $1.16 \mu \mathrm{m}$ obtained from the SEM image. The widths of peaks calculated by the double Gaussian fitting are 1.12 and $0.85 \mu \mathrm{m}$. Although the measured values are somewhat larger than the actual sizes, the two adjacent defects are sufficiently distinguishable, not only using the raw image of the transmitted $\mathrm{THz}$ amplitude but also by data analysis based on the double Gaussian fitting method.

In contrast, for $\mathrm{S} 2$, it is difficult to distinguish the double defects from the raw profiles shown in Figure $5 \mathrm{~b}, \mathrm{~d}$. The cross-sectional profile along the $x$-axis at the center of the amplitude peaks in Figure $5 \mathrm{~b}$ was also analyzed using the double Gaussian fitting method. The center-to-center distance and the widths of the peaks extracted by the fitting signals are 1.02, 1.09, and $0.70 \mu \mathrm{m}$, respectively. Despite the theoretical difficulty in distinguishing the two defects in S2 because of similar values of the widths of the peaks and the center-to-center distance, the discriminability between two adjacent defects can be increased by employing data analysis and suitable image processing methods.

The blind spot problem in the coherent system, finding the difference in thickness of materials by the phase change, occurs when the difference in optical path length from the surroundings matches the wavelength used in the system. The coherent $\mathrm{CW}-\mathrm{THz}$ system cannot distinguish bulk XLPE without any defect from defects occurring at a phase change of $2 \pi$. In that case, the transmitted $\mathrm{THz}$ waves can be undetectable. Therefore, to ensure the reliability of the measurements, it is necessary to verify that all defects of varying depths are measurable. We fabricated defects with $1.0 \mathrm{~mm}$ diameter and various depths, D, from 1.0 to $1.7 \mathrm{~mm}$ with a step size of $100 \mu \mathrm{m}$, and measured the samples at a frequency of $1 \mathrm{THz}$.

Based on the void defects with a refractive index of 1.0 and the XLPE material with a refractive index of approximately 1.5 , the optical path difference corresponding to a phase shift of $2 \pi$ at a frequency of $1 \mathrm{THz}$ will be $600 \mu \mathrm{m}$. There is no way to prevent void defects with depths equal to multiples of $600 \mu \mathrm{m}$ from being blind spots. Otherwise, the results shown in Figure 6 show that each defect is 
clearly identified, and only the amplitudes of the dips change slightly depending on D. In addition, the results do not include negative values, which are inevitably predicted to occur near the phase difference of $\pi$ in the coherent CW-THz imaging system.
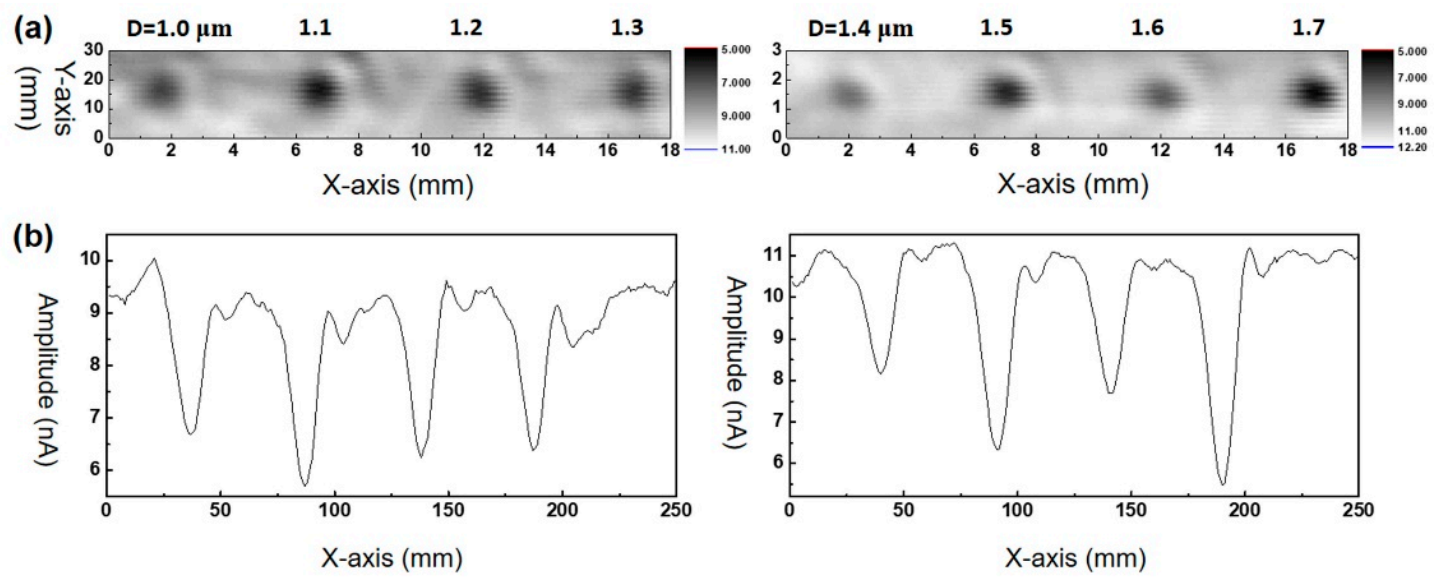

Figure 6. (a) Two-dimensional amplitude images of transmitted CW-THz waves for samples with various depths, D, starting from $1.0 \mathrm{~mm}$ and increasing up to $1.7 \mathrm{~mm}$ with a step size of $100 \mu \mathrm{m}$. The diameter of the artificial void defects is $1.0 \mathrm{~mm}$. The actual values of the depth may be slightly different from the designed parameters shown here. (b) Cross-sectional profiles along the $x$-axis at the center of the amplitude dips that originate from the defects shown in (a).

The fundamental mechanism of this phenomenon can be assumed to be related to the fact that the width of the beam waist of the $\mathrm{CW}-\mathrm{THz}$ waves at the focal point is larger than the diameter of the defects. In this case, even if the position of the defects is exactly at the focal point, part of the incident $\mathrm{THz}$ waves will pass through the surrounding area instead of the defect area. This effect manifests in the form of signal offsets of the transmission amplitudes. In addition, the THz wave incident on the artificial void defect, which can be classified as a subwavelength, cylindrical dielectric waveguide, experiences a different phase shift at each position. This may eliminate the phenomenon in which the measurement signal is not distinguished because of the blind spots. This means that general types of defects can be readily detected using the coherent $\mathrm{CW}-\mathrm{THz}$ imaging system.

\section{Conclusions}

We have demonstrated that artificial void defects located inside XLPE plates for power cable insulation can be detected using a CW-THz imaging system. Because the $\mathrm{THz}$ technique based on two photomixers serving as $\mathrm{THz}$ transmitter and receiver enables coherent detection, the change in phase that originates from the variation in optical path length of the $\mathrm{THz}$ waves caused by the void defects inside the XLPE plates was measured by the change in the amplitude of the transmitted $\mathrm{THz}$ waves. Two-dimensional images of the amplitudes of $\mathrm{THz}$ waves transmitted through single- or double-void defects with various diameters and gap widths show that internal defects of up to $0.5 \mathrm{~mm}$ size can be detected by the coherent CW-THz imaging system, which is a technique that can be used as an internal noncontact, nondestructive examination method. Limitations of the $\mathrm{CW} \mathrm{THz} \mathrm{imaging}$ system based on transmission geometry are that the samples containing the layers that strongly reflect or absorb the $\mathrm{THz}$ waves cannot be measured and the samples with the multilayered structures with uneven surfaces are hard to measure. However, such problems can be overcome by the applications of measurement systems combined with various geometries, such as reflection or scattering, and signal processing methods to remove signals due to other factors. Technical developments based on $\mathrm{CW}-\mathrm{THz}$ waves exhibit great potential in the context of various applications in the construction, automobile, manufacturing, electronics, energy, and electrical power industries, which require internal noncontact, nondestructive testing and inspection. 
Author Contributions: Conceptualization and methodology, J.W.L.; writing-original draft preparation, I.-S.L.; writing-review and editing, J.W.L.; data acquisition and analysis, I.-S.L. and J.W.L.; supervision, project administration, and funding acquisition, J.W.L. All authors have read and agreed to the published version of the manuscript.

Funding: This research was funded by the Korea Electric Power Corporation (Grant Number: R18XA06-79).

Acknowledgments: We acknowledge and thank J. H. Bang for the illustration in Figure 1a.

Conflicts of Interest: The authors declare no conflicts of interest.

\section{References}

1. Vahedy, V. Polymer insulated high voltage cables. IEEE Electr. Insul. Mag. 2006, 22, 13-18. [CrossRef]

2. Khalil, M.S. International research and development trends and problems of HVDC cables with polymeric insulation. IEEE Electr. Insul. Mag. 2002, 13, 35-47. [CrossRef]

3. Motori, A.; Sandrolini, F.; Montanari, G.C. A contribution to the study of aging of XLPE insulated cables. IEEE Trans. Power Deliv. 1991, 6, 34-42. [CrossRef]

4. Gulmine, J.V.; Akcelrud, L. Correlations between structure and accelerated artificial ageing of XLPE. Eur. Polym. J. 2006, 42, 553-562. [CrossRef]

5. Hozumi, N.; Takeda, T.; Suzuki, H.; Okamoto, T. Space charge behavior in XLPE cable insulation under 0.2-1.2 MV/cm dc fields. IEEE Trans. Dielectr. Electr. Insul. 1998, 5, 82-90. [CrossRef]

6. Lee, S.Y.; Kim, Y.H.; Lee, I.H.; Amyot, N. Influence of internal residual mechanical stresses on local dielectric strength of EHV extruded XLPE cables. In Proceedings of the Electrical Insulation Conference and Electrical Manufacturing and Coil Winding Conference, Cincinnati, OH, USA, 18 October 2001; pp. 345-348.

7. Boukezzi, L.; Boubakeur, A.; Lallouani, M. Effect of artificial thermal aging on the crystallinity of XLPE insulation cables: X-ray study. In Proceedings of the 2007 IEEE International Conference on Electrical Insulation and Dielectric Phenomena (CEIDP), Vancouver, BC, Canada, 14-17 October 2007; p. 65.

8. Wenbin, S.; Ju, T.; Cheng, P.; Guodong, M.; Zhang, M. Improvement of insulation defect identification for DC XLPE cable by considering PD aging. Int. J. Electr. Power 2020, 114, 105409.

9. Lee, I.S.; Lee, J.W. Effects of thermal aging on cellulose pressboard using terahertz time-domain spectroscopy. Curr. Appl. Phys. 2019, 19, 1145-1149. [CrossRef]

10. Kang, S.B.; Kim, W.S.; Chung, D.C.; Joung, J.M.; Kwak, M.H. Degradation diagnosis of transformer insulating oils with terahertz time-domain spectroscopy. J. Korean Phys. Soc. 2017, 71, 986-992. [CrossRef]

11. Wang, L.; Tang, C.; Zhu, S.; Zhou, S. Terahertz time domain spectroscopy of transformer insulation paper after thermal aging intervals. Materials 2018, 11, 2124. [CrossRef] [PubMed]

12. Mittleman, D.M.; Gupta, M.; Neelamani, R.; Baraniuk, R.G.; Rudd, J.V.; Koch, M. Recent advances in terahertz imaging. Appl. Phys. B 1999, 68, 1085-1094. [CrossRef]

13. Ferguson, B.; Zhang, X.C. Materials for terahertz science and technology. Nat. Mater. 2002, 1, $26-33$. [CrossRef] [PubMed]

14. Seo, M.A.; Lee, J.W.; Kim, D.S. Dielectric constant engineering with polymethylmethacrylate-graphite metastate composites in the terahertz region. J. Appl. Phys. 2006, 99, 066103. [CrossRef]

15. Yan, Z.; Shi, W.; Hou, L.; Xu, M.; Yang, L.; Dong, C.; Li, S. Investigation of aging effects in cross-linked polyethylene insulated cable using terahertz waves. Mater. Res. Express 2017, 4, 015304. [CrossRef]

16. Zhang, Y.; Lian, Z.; Li, J.; Li, S. Terahertz time-domain spectroscopy characterization of aged XLPE cable insulation. In Lecture Notes in Electrical Engineering, Proceedings of the 21st International Symposium on High Voltage Engineering, Budapest, Hungary, 26-30 August 2019; Springer: Cham, Switzerland, 2019; Volume 589, pp. 878-886.

17. Komatsul, M.; Ohkil, Y.; Mizun, M.; Fukunaga, K. Terahertz spectroscopic observation of cross-linked polyethylene aged simultaneously by heat and gamma-rays. In Proceeding of the 2012 IEEE 10th International Conference on the Properties and Applications of Dielectric Materials, Bangalore, India, 24-28 July 2012.

18. Ohki1, Y.; Komatsu, M.; Mizuno, M.; Fukunaga, K. Terahertz spectroscopy as a novel method for diagnosing the integrity of polymer insulated cables. In Proceeding of the 2012 Annual Report Conference on Electrical Insulation and Dielectric Phenomena, Montreal, QC, Canada, 14-17 October 2012; pp. 203-206.

19. Boone, K.G.; Holder, D.S. Electrical impedance tomography method and apparatus. U.S. Patent No. 5,919,142, 6 July 1999. 
20. James, R.; Faisal Haider, M.; Giurgiutiu, V.; Lilienthal, D. A simulative and experimental approach toward Eddy current nondestructive evaluation of manufacturing flaws and operational damage in CFRP composites. J. Nondestruct. Eval. 2020, 3, 011002.

21. Jhang, K.Y. Nonlinear ultrasonic techniques for nondestructive assessment of micro damage in material: A review. Int. J. Precis. Eng. Man. 2009, 10, 123-135. [CrossRef]

22. Schmidt, V.; Husinsky, W.; Betz, G. Dynamics of laser desorption and ablation of metals at the threshold on the femtosecond time scale. Phys. Rev. Lett. 2000, 85, 8516. [CrossRef] [PubMed]

(C) 2020 by the authors. Licensee MDPI, Basel, Switzerland. This article is an open access article distributed under the terms and conditions of the Creative Commons Attribution (CC BY) license (http://creativecommons.org/licenses/by/4.0/). 